\title{
Appropriateness of antimicrobial therapy: a multicentre prevalence survey in the Netherlands, 2008-2009
}

I Willemsen ${ }^{1}$, T van der Kooij², B van Benthem², J Wille3, J Kluytmans (jankluytmans@gmail.com)1,4

1. Laboratory for Microbiology and Infection Control, Amphia Hospital, Breda, the Netherlands

2. Netherlands Centre for Infectious Disease Control, National Institute for Public Health and the Environment (Rijksinstituut voor Volksgezondheid en Milieu, RIVM), Bilthoven, the Netherlands

3. Dutch Institute for Healthcare Improvement (CBO), Utrecht, the Netherlands

4. Department of Microbiology and Infection Control, VU University Medical Center (VU medisch centrum, VUmc), Amsterdam, the Netherlands

Citation style for this article:

Willemsen I, van der Kooij T, van Benthem B, Wille J, Kluytmans J. Appropriateness of antimicrobial therapy: a multicentre prevalence survey in the Netherlands, 2008-2009. Euro Surveill. 2010;15(46):pii=19715. Available online: http://www.eurosurveillance.org/ViewArticle.aspx?Articleld=19715

Article published on 18 November 2010

A survey was carried out to determine the prevalence and appropriateness of antimicrobial therapy (AMT) in the Netherlands and to identify determinants for inappropriate AMT. Prevalence surveys of patients hospitalised in the Netherlands were performed three times in 2008 and 2009. Patients' demographic, infectionrelated and AMT-related data were collected from hospital wards. A total of 19 hospitals participated, consisting of a mix of university, teaching and general hospitals, which were distributed evenly across the country. The appropriateness of AMT was assessed using a standardised algorithm based on local AMT prescription guidelines. A total of 7,853 patients were included, of which 2,327 (29.6\%) patients were on AMT (range: $20.8-39.5 \%$ ). In 372 patients (16\% of patients on AMT), treatment was considered inappropriate. In $265(11.4 \%)$ patients on AMT, appropriateness of treatment was not judged because of insufficient information. The percentage of patients without a judgment varied considerably between the participating hospitals (range: $1.3-36.2 \%$ ). Appropriate AMT use was significantly associated with a patient being in an intensive care unit, having a central venous catheter and being given beta-lactamase-sensitive penicillins. The use of fluoroquinolones was significantly associated with more frequent inappropriate use. There was considerable and significant variation between the participating hospitals in the amount of antimicrobials prescribed and the appropriateness of their use. To improve the completeness and reliability of such surveys, there is a need for intensive training of observers and medical staff in recording information.

\section{Introduction}

Point prevalence surveys are useful ways of investigating healthcare-related events, including antimicrobial use. The first report on antimicrobial use measured in prevalence surveys was published in 1983 [1]. More recently a European project - the European Surveillance of Antimicrobial Consumption (ESAC) - has standardised a method to determine the prevalence of antimicrobial therapy (AMT) in hospitals [2]. In a previous study, performed in a teaching hospital in the Netherlands, we showed that besides the prevalence of AMT, the appropriateness of AMT for individual patients could also be determined, basing the judgement on local antibiotic prescription guidelines [3]. This enables researchers to quantify the number of patients who are treated even when treatment is not indicated or who are treated with a drug that is not the preferred choice. In addition, it was possible to identify determinants of inappropriate use of AMT [3]. The objective of the current study was to determine whether prevalence studies could be used in other hospitals as well and whether they could also be used as a tool for benchmarking.

The study was coordinated by the PREZIES (Prevention of Nosocomial Infections by Surveillance) network a collaboration between participating hospitals, the Dutch Institute for Healthcare Improvement (CBO) and the National Institute for Public Health and the Environment (Rijksinstituut voor Volksgezondheid en Milieu, RIVM), RIVM).

\section{Methods}

Prevalence surveys

Hospitals were recruited from PREZIES. Those that had already participated in prevalence surveys for nosocomial infections were invited to add AMT use to this survey. Participation was on a voluntary basis.

Three prevalence surveys of hospitalised patients were performed in spring 2008, autumn 2008 and spring 2009. All patients that were present in the hospital at 00:01 on the day of the survey were included. Patients in day care (including haemodialysis patients) and psychiatric wards were excluded. Data from the hospital wards were collected by infection control practitioners. A standardised case record form, to document patients' demographic, infection-related and AMT-related data, was used. The infection control practitioners received 
training during workshops on how to collect the data and how to judge the appropriateness of AMT. The following demographic variables were recorded: age, sex, medical speciality, type of ward and presence of infection on admission. Nosocomial infections were recorded using the definitions from the United States Centers for Disease Control and Prevention (CDC) and patients had to be symptomatic or still being treated on the day of the survey $[4,5]$. Furthermore, the use of antimicrobial agents and dosage were noted. If more than one antimicrobial was prescribed for one patient, all antibiomicrobials, up to a maximum of three, were registered. Antifungal and antiviral therapy as well as medication for tuberculosis were excluded from the study. The main reasons were that not all local guidelines had specific recommendations for these agents and susceptibility of pathogens to these agents was not always determined by the local microbiology laboratories.

\section{Appropriateness of antimicrobial therapy \\ The appropriateness of AMT was determined using a standardised method developed by Gyssens et al. [6], using the following classifications: correct decision, incorrect decision, incorrect choice or insufficient data. This classification system obviously only takes into account patients that are on AMT. However, using our approach it is possible to examine the appropriateness of not prescribing AMT also, as described in our previ- ous study [3]. A correct decision was deemed appro- priate; incorrect decision and incorrect choice were considered inappropriate (the evaluation criteria are summarised in Table 1 ).}

The appropriateness of AMT was judged according to the local AMT prescription guidelines present in all

\section{TABLE 1}

Evaluation criteria for appropriateness of antimicrobial therapy, the Netherlands, 2008-2009

\begin{tabular}{|l|}
\hline Categories and criteria \\
\hline 1. Correct decision (appropriate use) \\
\hline 1.1 No AMT and no infection and no AMT needed \\
\hline 1.2 No AMT and infection and no AMT needed \\
\hline $\begin{array}{l}\text { 1.3 AMT and infection and appropriate choice and appropriate } \\
\text { use }\end{array}$ \\
\hline 2. Incorrect decision (inappropriate use) \\
\hline 2.1 No AMT and infection and AMT needed \\
\hline 2.2 AMT and no infection and no prophylaxis and no AMT needed \\
\hline 2.3 AMT and no infection and prophylaxis and no AMT needed \\
\hline 3. Incorrect choice (inappropriate use) \\
\hline 3.1 Divergence from guidelines \\
\hline 4. Missing data (insufficient information) \\
\hline $\begin{array}{l}\text { 4.1 No AMT and not enough diagnostic information about } \\
\text { infection }\end{array}$ \\
\hline $\begin{array}{l}\text { 4.2 Infection and not enough diagnostic information if AMT is } \\
\text { needed }\end{array}$ \\
\hline 4.3 AMT and not enough diagnostic information about infection \\
\hline 4.4 Infection and not enough information about AMT \\
\hline
\end{tabular}

AMT: antimicrobial therapy. participating hospitals. These local guidelines are based on the national policy developed by the Dutch Working Party on Antibiotic Policy (Stichting Werkgroep Antibiotica Beleid, SWAB) [7,8]. The infection control practitioners assessed the appropriateness of AMT initially: if they could not decide, a consultant microbiologist or infectious disease physician made the final judgment. The consultant microbiologist or infectious disease physician also judged all patients in intensive care units, all patients who received AMT without having an active infection (according to the survey), all patients who did not receive AMT and did have an active infection and all patients who received AMT that was not according to the local AMT guidelines.

If all the antimicrobial agents that a patient received were considered correct, the treatment was considered appropriate. If one or more of the antimicrobial agents was considered incorrect, the treatment was considered inappropriate. If it was not possible to decide whether use of a particular antimicrobial agent was correct due to incomplete information, treatment was recorded as insufficient information. We did not assess the reproducibility of the judgments.

Data analysis, quality control

and statistical analysis

Privacy of patients is ensured by decoding all data, as required by the privacy regulations in the Netherlands. Data were entered in the PREZIES database or a hospital-owned database and subsequently coded and transferred to PREZIES.

Data were analysed using SPSS version 17.0. Treatment for which there was insufficient information was recorded as a missing value. Categorical variables were analysed by Fisher's exact test or chi-square test where appropriate: continuous variables were analysed using a t-test or Mann-Whitney U test where appropriate. Binary logistic regression analysis was performed: all variables with a $p$ value less than 0.1 in univariate analyses were entered into the multivariate model. Statistical significance was accepted when the chance for coincidence was less than $5 \%$. Finally, a sensitivity analysis was performed. In this, the univariate and multivariate analyses were repeated: once categorising AMT use as appropriate for all patients for whom AMT use could not be judged and once categorising it as inappropriate.

\section{Results}

A total of 7,853 patients were included, from 19 hospitals. They were a mix of university, teaching and general hospitals, which were distributed evenly across the country. Of these, 13 participated in one of the three surveys, five participated in two surveys and one participated in all three. A mean of 302 patients were included per hospital per prevalence survey (range: 103-552; standard deviation: 149). 
Patient characteristics and

nosocomial infections

Overall 3,784 (48.2\%) patients were male, and the mean age was 62 years (median: 67 years). On the day of the survey 426 patients (5.4\%) had at least one active nosocomial infection.

\section{Antimicrobial therapy}

A total of 2,327 patients (29.6\%) were on AMT (range: 20.8-39.5\%). The mean prevalence of AMT per participating hospital is shown in Figure 1.

Of the 2,327 patients on AMT, $433(18.6 \%)$ were treated with two antimicrobials, and $58(2.5 \%)$ were treated with three or more. In total 2,876 courses of antimicrobial agents were administered, of which 1,709 (59.4\%) were given intravenously (range: 42.2-75.9\%).

The first antimicrobial agent was considered appropriate in $1,690(72.6 \%)$ patients. In $149(6.4 \%)$ patients the first antimicrobial agent was considered not justified

\section{FIGURE 1}

Mean prevalence of antimicrobial therapy per participating hospital, the Netherlands, 2008-2009

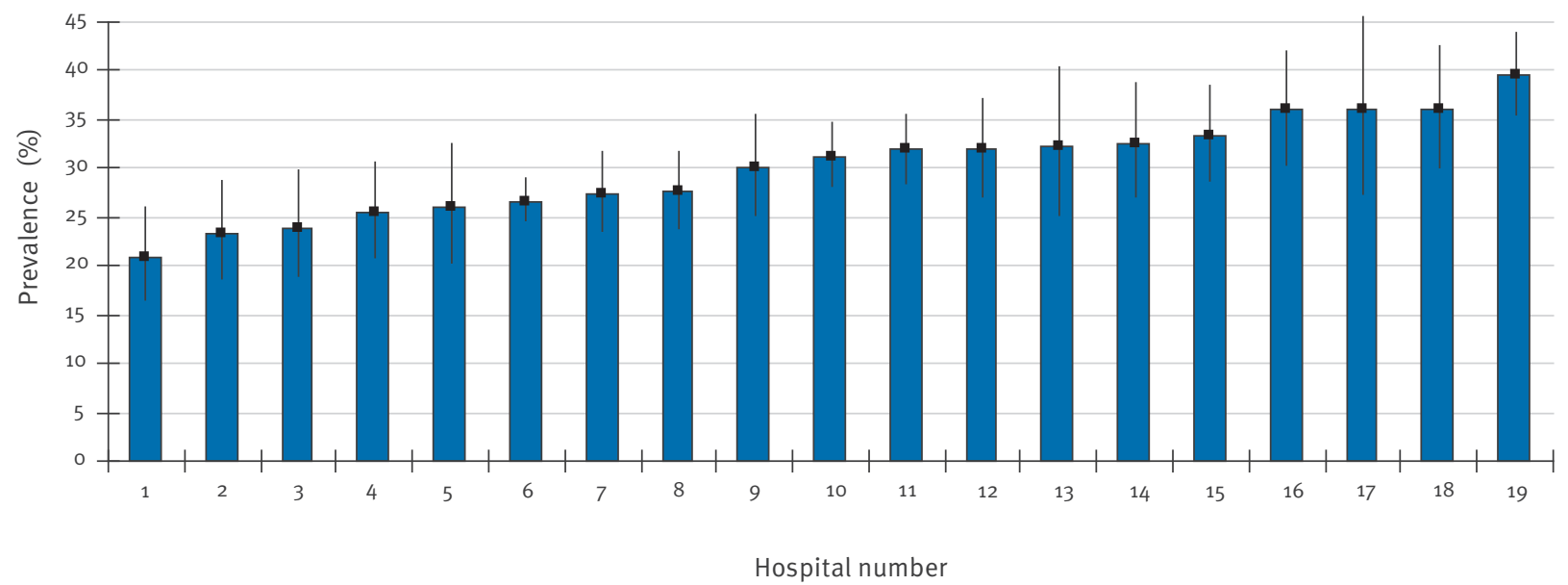

The hospitals are shown in increasing order of prevalence. Vertical bars represent the $95 \%$ confidence intervals.

\section{FIGURE 2}

Inappropriate antimicrobial therapy and proportion of patients without antimicrobial therapy judgment ${ }^{\mathrm{a}}$, by participating hospital, the Netherlands, 2008-2009

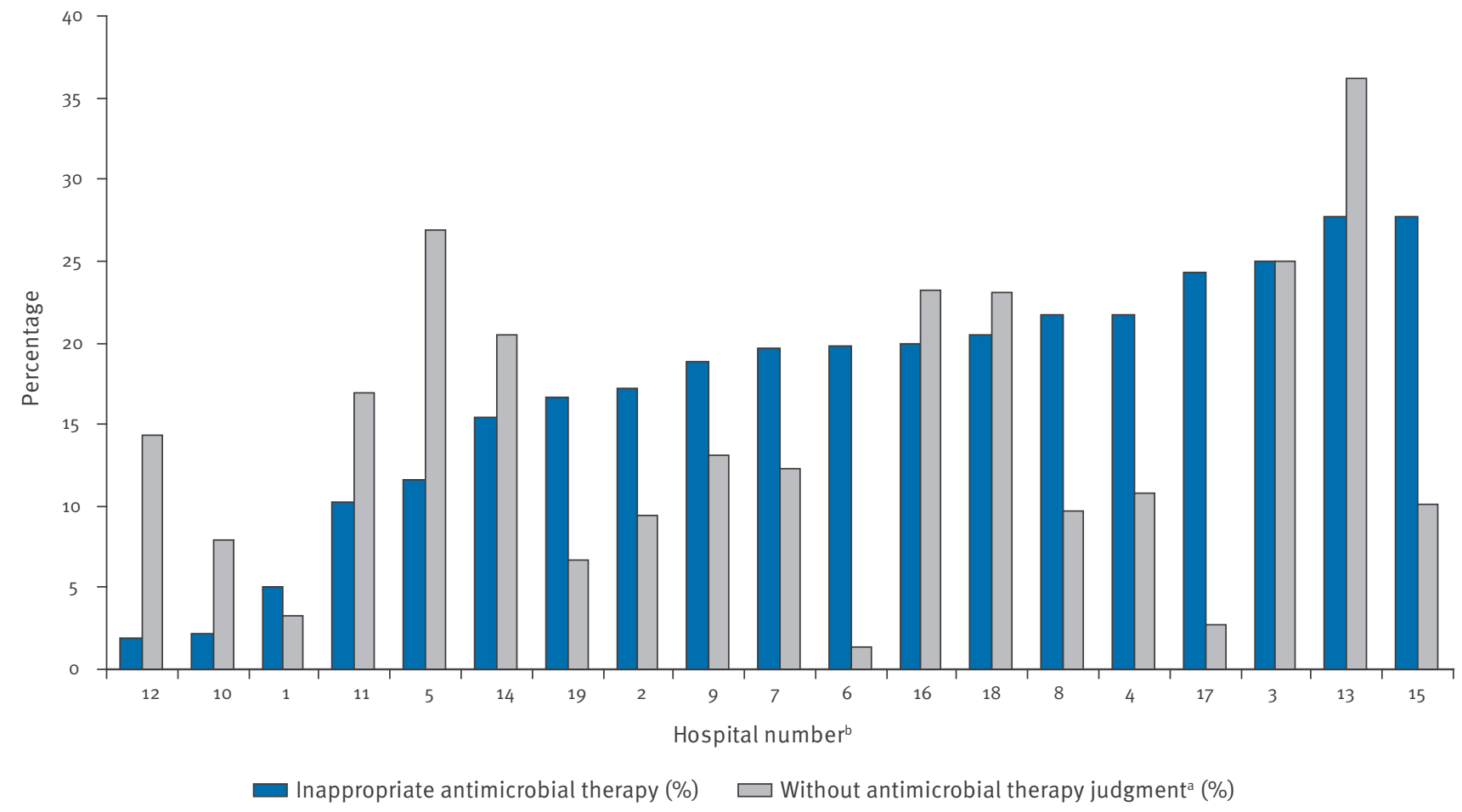

The hospitals are shown in order of increasing proportion of inappropriate use.

a Due to missing information.

${ }^{\mathrm{b}}$ The numbering of hospitals is identical to the hospital numbers in Figure 1. 
and therefore inappropriate. In 223 (9.6\%) patients AMT was justified, but the choice of the agent was not according to the guidelines. In 265 (11.4\%) patients no decision was made due to insufficient information. The second antimicrobial agent was considered appropriate in 384 patients $(78.2 \%$ of the 491 patients treated with more than one antimicrobial agent), not justified in $26(5.3 \%)$ and justified but an incorrect choice in $39(7.9 \%)$ patients. In $42(8.6 \%)$ patients no choice was made due to insufficient information. The third antimicrobial was considered appropriate in 50 of the 58 patients treated with at least three antimicrobial

TABLE 2

Use of antimicrobial agents in participating hospitals, the Netherlands, 2008-2009

\begin{tabular}{|c|c|c|c|c|c|c|c|c|}
\hline \multirow{2}{*}{ Antimicrobial agent } & \multicolumn{2}{|c|}{ First antibiotic } & \multicolumn{2}{|c|}{ Second antibiotic } & \multicolumn{2}{|c|}{ Third antibiotic } & \multicolumn{2}{|c|}{ Total } \\
\hline & $\mathrm{n}$ & $\%$ & $\mathrm{n}$ & $\%$ & $\mathrm{n}$ & $\%$ & $n$ & $\%$ \\
\hline beta-lactamase-sensitive penicillins & 58 & 2.5 & 12 & 2.4 & 1 & 1.7 & 71 & 2.5 \\
\hline beta-lacatamase-resistant penicillins & 150 & 6.4 & 17 & 3.5 & 1 & 1.7 & 168 & 5.8 \\
\hline Carbapenems & 44 & 1.9 & 5 & 1.0 & 2 & 3.4 & 51 & 1.8 \\
\hline Co-amoxicillin-clavulanic acid & 715 & 30.7 & 42 & 8.6 & & & 757 & 26.3 \\
\hline First-generation cephalosporins & 124 & 5.3 & 2 & 0.4 & 1 & 1.7 & 127 & 4.4 \\
\hline Second-generation cephalosporins & 147 & 6.3 & 35 & 7.1 & 2 & 3.4 & 184 & 6.4 \\
\hline Third- and fourth-generation cephalosporins & 177 & 7.6 & 30 & 6.1 & 4 & 6.9 & 211 & 7.3 \\
\hline Co-piperacillin-tazobactam & 63 & 2.7 & 7 & 1.4 & 3 & 5.2 & 73 & 2.5 \\
\hline Sulfonamides and trimethoprim & 107 & 4.6 & 15 & 3.1 & 3 & 5.2 & 125 & 4.3 \\
\hline Fluoroquinolones & 303 & 13.0 & 93 & 18.8 & 8 & 13.8 & 404 & 14.0 \\
\hline Glycopeptides & 37 & 1,6 & 16 & 3,3 & 2 & 3,4 & 55 & 1,9 \\
\hline Imidazole derivates & 55 & 2,4 & 68 & 13,9 & 4 & 6,9 & 127 & 4,4 \\
\hline Lincosamides and macrolides & 84 & 3,6 & 39 & 8,0 & 10 & 17,2 & 133 & 4,6 \\
\hline Broad-spectrum penicillin & 128 & 5,5 & 34 & 6,9 & 2 & 3,4 & 164 & 5,7 \\
\hline Aminoglycosides & 30 & 1,3 & 40 & 8,2 & 8 & 13,8 & 78 & 2,7 \\
\hline Tetracyclines & 42 & 1,8 & 6 & 1,2 & 1 & 1,7 & 49 & 1,7 \\
\hline Other antimicrobials & 63 & 2,7 & 30 & 6,1 & 6 & 10,3 & 99 & 3,4 \\
\hline Total & 2,327 & 81 & 491 & 17 & 58 & 2 & $2,876^{a}$ & 100 \\
\hline
\end{tabular}

a Total number of courses of antimicrobial agents administered that were recorded on the survey days.

\section{FIGURE 3}

Relative risk for inappropriate use of antimicrobial therapy by group of antimicrobial agent ${ }^{\mathrm{a}}$, the Netherlands, 2008-2009

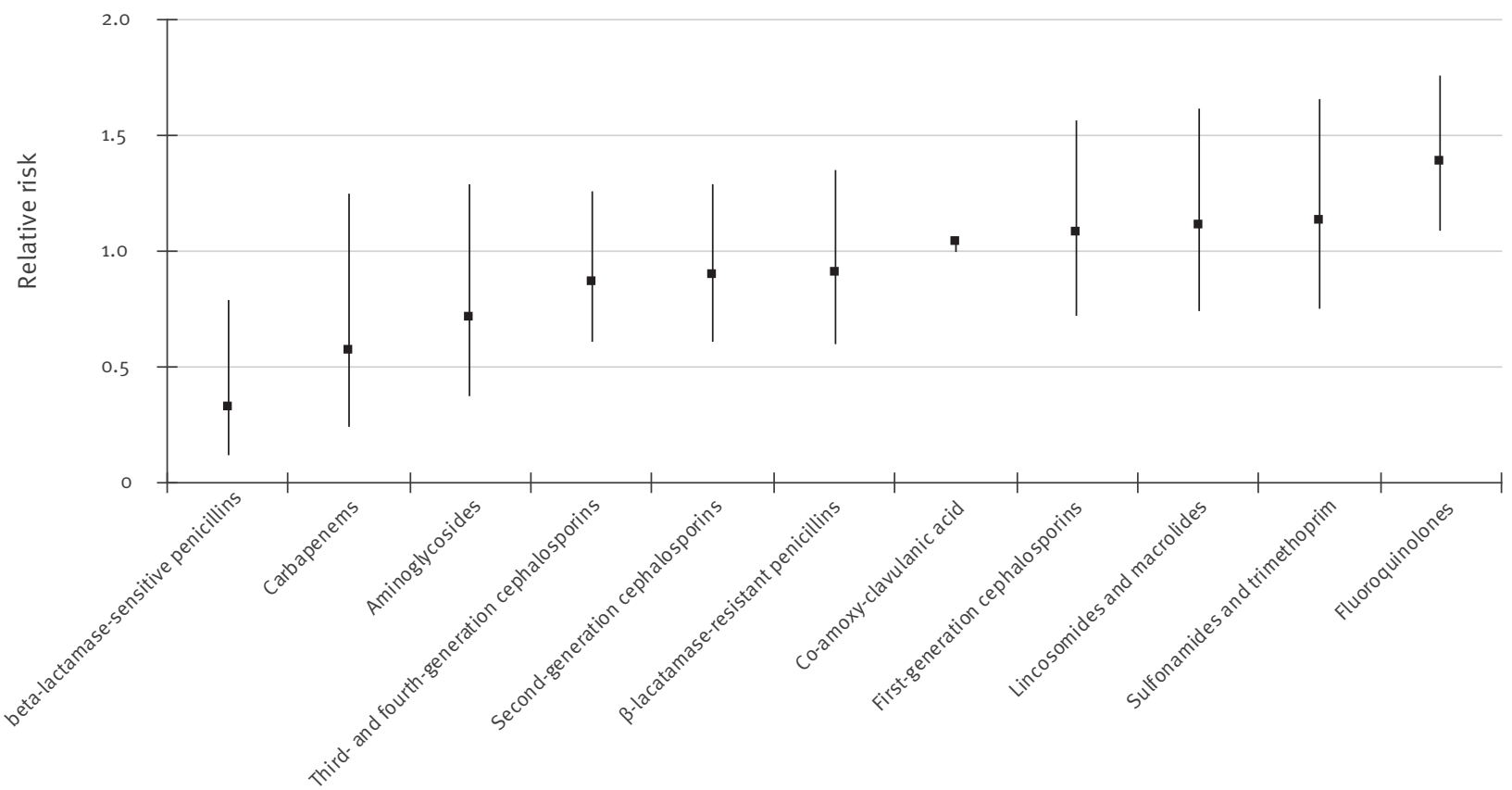


agents, not justified in two and justified but an incorrect choice in two patients. Four were not judged due to insufficient information.

Judgment of the appropriateness of AMT per patient showed that 372 patients ( $16 \%$ of the patients on AMT; $4.7 \%$ of the total population) were treated inappropriately. Figure 2 shows the variations in the proportion of AMT considered inappropriate in the different hospitals (range: $5.0-32.4 \%$ ).

For 265 patients (11.4\%) on AMT it was not possible to judge appropriateness because of insufficient information. Figure 2 shows the variations in the proportion of patients on AMT who could not be judged in the participating hospitals (range: $1.3-36.2 \%$ ).

Of the patients who did not receive AMT $(n=5,526), 945$ were not judged for the appropriateness of the decision not to treat. This was mainly due to four hospitals that did not judge patients who were not receiving AMT. Of the 4,581 patients not receiving AMT who were judged, the decision not to use AMT was considered appropriate for 4,497 (98.2\%) patients. For 22 patients (0.5\% of those not on AMT who were judged), patients did not receive $A M T$, although this was indicated. For 62 (1.4\%) patients not on AMT it was not possible to assess the appropriateness because of insufficient information.

\section{FIGURE 4}

Relative risk for inappropriate use of antimicrobial therapy by medical specialty ${ }^{\text {a }}$, the Netherlands, 2008-2009

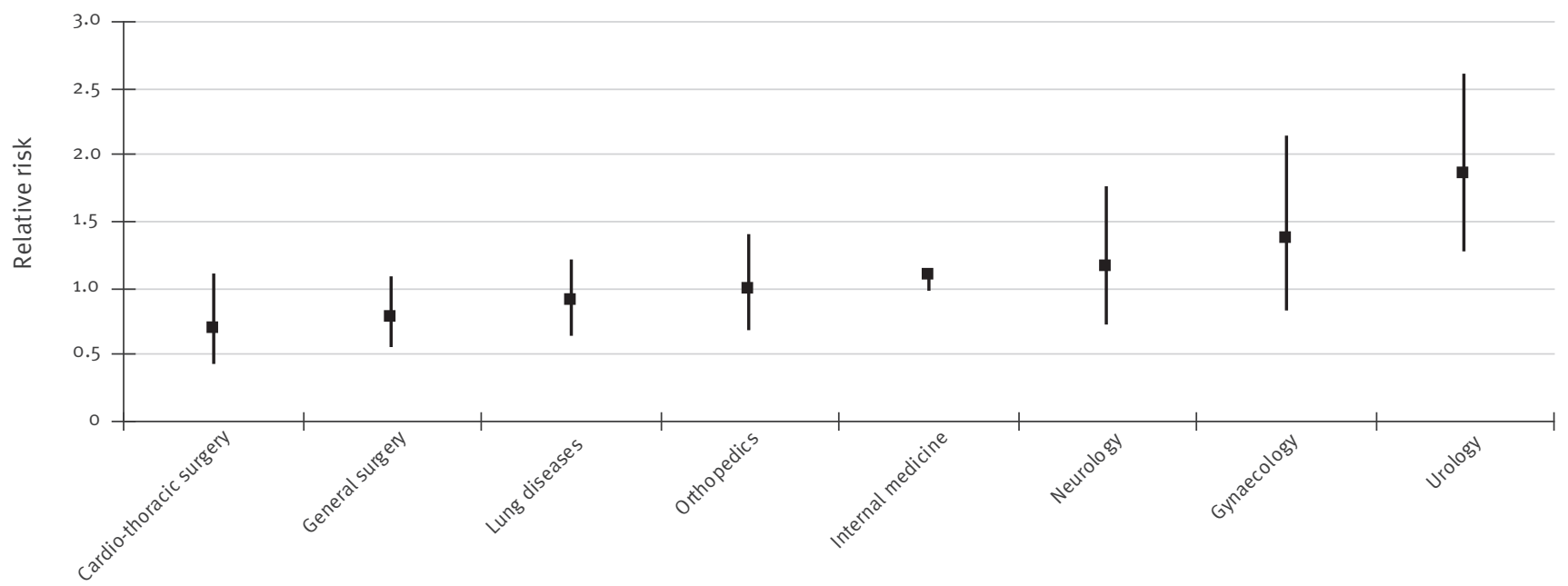

Vertical bars represent the $95 \%$ confidence intervals.

a Internal medicine as reference.

\section{FIGURE 5}

Relative risk for inappropriate use of antimicrobial therapy ${ }^{\mathrm{a}}$, the Netherlands, 2008-2009

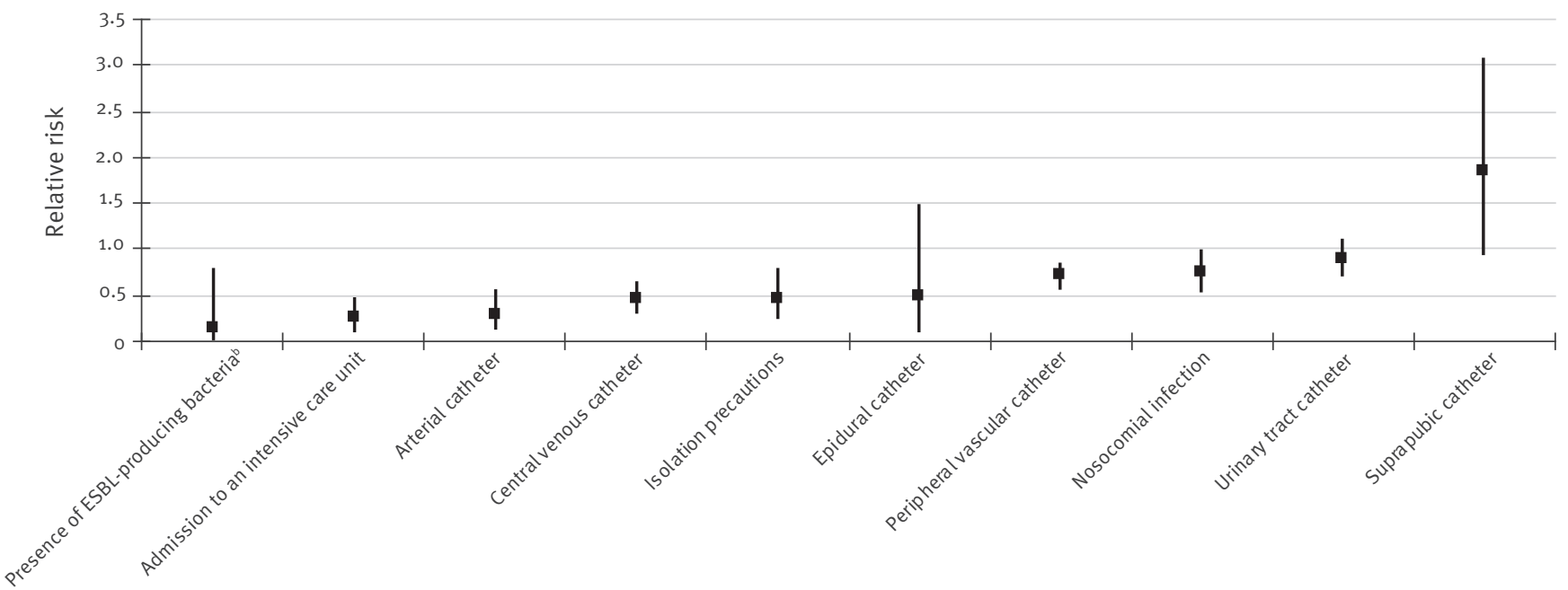

Vertical bars represent the $95 \%$ confidence intervals.

a All determinants are dichotomous variables, which are compared to their counterpart.

${ }^{\mathrm{b}}$ Extended-spectrum beta-lactamase-producing Gram-negative rods. 
Table 2 shows the distribution of the use of various antimicrobials. Co-amoxicillin-clavulanic acid was most commonly used, second were the fluoroquinolones and the third were the third- and fourth-generation cephalosporins.

\section{Determinants of inappropriate}

\section{use of antimicrobial therapy}

In the univariate analysis, use of fluoroquinolones was significantly associated with more frequent inappropriate use of AMT (relative risk: 1.4). The use of beta-lactamase-sensitive penicillins was significantly associated with more frequent appropriate use of AMT (relative risk: 0.3) (Figure 3).

Considering the use of AMT in the different medical specialties, urology $(p=0.002)$ proved to be significantly associated with more frequent inappropriate use (Figure 4). None of the specialties was significantly associated with more frequent appropriate use.

Figure 5 shows that the presence of a suprapubic catheter was significantly associated with more frequent inappropriate use (relative risk: 1.9). The following factors were associated with more frequent appropriate use of AMT: having a central venous catheter, a peripheral vascular catheter or an arterial catheter, the presence of ESBL (extended-spectrum beta-lactamaseproducing bacteria, being admitted to an intensive care unit, being in isolation precautions and having a nosocomial infection.

In multivariate analyses, taking the effects of all the above-mentioned variables into account, we found that the hospitals themselves were important determinants associated with appropriate or inappropriate use of AMT (Table 3). Furthermore, increasing age $(p=0.024)$, being in an intensive care unit $(p=0.002)$, having a central venous catheter $(p=0.12)$, peripheral vascular catheter $(p=0.005)$ and nosocomial infection $(p=0.049)$ and use of beta-lactamase-sensitive penicillins $(p=0.017)$ were significantly associated with appropriate use in multivariate analyses. The presence of a suprapubical catheter $(p=0.017)$ or the use of fluoroquinolones ( $p<0.001)$ were significantly associated with inappropriate use of AMT. No colinearity was found between the variables in the multivariate model.

\section{Discussion and conclusions}

The mean prevalence of AMT in this study was $29.6 \%$ (range: $20.8-29.5 \%$ ). The most recent study that can be used for comparison is from the European Surveillance of Antimicrobial Consumption (ESAC) which found a similar prevalence of 30\% (range: 19 $-59 \%$ ) in 20 European hospitals in 2006 [2]. Other prevalence studies in hospitals in the United Kingdom and Turkey also showed similar rates of antimicrobial use [9-11]. Although the overall prevalence in our study is comparable to that of other large surveys, there were large variations between the participating hospitals. This range of appropriate AMT use can be explained by differences in the patient populations and by differences in prescription policies between hospitals and between individual prescribers.

Of all patients on AMT in this study, the use was considered inappropriate in 372 (16\%; range: $1.9-27.7 \%$ ). The patients concerned comprised $4.7 \%$ of the total number of patients, which may seem relatively unimportant. However, this means that annually approximately 10,000 days of unjustified AMT are given in a hospital with 200,000 patient days a year. Treating patients with AMT when such treatment is not indicated is known to be associated with higher costs, more side effects and more antimicrobial resistance $[12,13]$.

Our study showed that the proportion of patients for whom AMT was judged to be inappropriate varied between hospitals. AMT use could not be judged for 265 patients due to insufficient information. Deciding on the appropriateness of AMT use is often not easy. However, the difference between the hospitals is remarkable. The hospitals with the lowest proportion of cases that could not be judged were hospitals with previous experience with this kind of survey. Possibly these kinds of judgments require more extensive training. During a session that was organised with the infection control practitioners and consultant microbiologists to discuss the findings, it was thought that more training and discussion of difficult cases in the study group would probably result in a reduction of the number of cases that could not be judged.

Our study showed that the participating hospital is a determinant itself and had a great influence in the analyses of determinants associated with inappropriate or appropriate AMT use. We were unable to identify specific characteristics of the hospitals that were responsible for more frequent inappropriate use. Nevertheless, use of fluoroquinolones proved to be a significant risk factor for inappropriate use of AMT. Fluorouinolones were the second most frequently used antimicrobials. The ESAC reported that use of fluoroquinolones increased most rapidly of all groups of antimicrobial agents, with a rise of $15 \%$ or more from 2000 to 2005 in almost half of all participating countries [14]. At the same time antimicrobial resistance against the fluoroquinolones increased from $5 \%$ (in 2001) to $14 \%$ (in 2008) in Escherichia coli and from 4\% (in 2005) to $8 \%$ (in 2008) in Klebsiella pneumoniae [15]. This highlights the importance of undertaking targeted interventions to reduce inappropriate use of fluoroquinolones. Data from prevalence surveys such as those described here provide support for such action.

Other determinants associated with a more appropriate use of AMT were variables associated with the clinical complexity of the patients (e.g. being admitted to an intensive care unit and having a central venous catheter). In the Netherlands, a microbiologist or infectious disease physician is almost always consulted in the assessment of these complicated cases. However, 
the largest group of patients being treated with antibiotics comprises relatively uncomplicated cases: most of these are not monitored by the microbiologist or infectious disease physician. A prevalence survey does include this group of patients and delivers information on the appropriateness of use. In our experience, it is this group in which a substantial improvement of the quality of antibiotic prescription can be achieved.

The fact that the treatment or lack of treatment of some patients could not be judged may have affected the outcome of our study. However, a sensitivity analysis showed that this did not affect the conclusions about the appropriateness of AMT use in the participating hospitals. We did not collect information on what factors in the hospitals with higher inappropriate use may have contributed to this. Further studies are warranted, since they may offer clues for further improvement.

In this study we identified those patients who inadvertently did not receive AMT (22 patients, $0.3 \%$ of total study population). There was no further analysis of the 22 patients. In an earlier single-centre study, we found a similar fraction of such patients ( 25 patients, $0.6 \%$ of total study population); further investigation showed that those patients were not adversely affected at discharge [3].

The extent of intravenous administration of antimicrobials $(59.4 \%)$ suggests that there is room for improvement. Intervention studies performed in the Netherlands showed that intravenous administration can be reduced relatively easily by targeted interventions $[16,17]$. A switch to oral therapy often results in a shorter hospital admission. In our study, the appropriateness of the route of administration was not assessed

In conclusion, we have demonstrated that it is possible to collect prevalence data on use of AMT at a national level. Individual hospital data can be very helpful in initiating targeted interventions to improve AMT use [17]. However, in order to produce more reliable results of such surveys, the number of patients for whom the appropriateness of AMT use could not be judged has to be reduced. Therefore training of infection control practitioners and consultant microbiologists has to be intensified and medical staff need to be trained in how to record information, in order to get an unambiguous assessment of use of AMT.
4. Horan TC, Gaynes RP, Martone WJ, Jarvis WR, Emori TG. CDC definitions of nosocomial surgical site infections, 1992: a modification of CDC definitions of surgical wound infections. Infect Control Hosp Epidemiol. 1992;13(10):606-8.

5. Horan TC, Gaynes RP. Surveillance of nosocomial infections. In: Mayhall CG, editor. Hospital epidemiology and infection control. Philadelphia: Lippincott Williams \& Wilkins: 2004. p. 1659-1702.

6. Gyssens IC, van den Broek PJ, Kullberg B, Hekster YA, van der Meer JWM. Optimizing antimicrobial therapy. A method for antimicrobial drug use evaluation. I Antimicrob Chemother. 1992;30:724-7.

7. Van Kasteren MEE, Wijnands WJ, Stobberingh EE, Janknegt $R$, Verbrugh HA, van der Meer JW. [Optimizing antibiotics use policy in the Netherlands. I. The Netherlands Antibiotics Policy Foundation (SWAB)]. Ned Tijdschr Geneeskd. 1998;142:949-51.

8. Dutch Working Party on Antibiotic Policy (SWAB). Nethmap 2008 - Consumption of antimicrobial agents and antimicrobial resistance among medically important bacteria in the Netherlands. Amsterdam: SWAB; 2008. Available from: http:// www.swab.nl/swab/swabcms.nsf/(WebFiles)/E32F6709B7DB7F 2EC125744Fo02ACAA5/\$FILE/NethMap_2008.pdf

9. Seaton RA, Nathwani D, Burtyon P, McLaughlin C, MacKenzie AR, Dundas S, Ziglam H, Gourlay Y, Beard K, Douglas E. Point prevalence survey of antibiotic use in Scottish hospitals utilising the Glasgow Antimicrobial Audit Tool (GAAT). Int J Antimicrob Agents. 2007;29(6):693-9.

10. Usluer G, Ozgunes I, Leblebicioglu H, Turkish Antibiotic Utilization Study Group. A multicenter point-prevalence study: antimicrobial prescription frequencies in hospitalized patients in Turkey. Ann Clin Microbiol Antimicrob. 2005;4:16.

11. Ang L, Laskar R, Gray JW. A point prevalence study of infection and antimicrobial use at a UK children's hospital. J Hosp Infect. 2008;68(4):372-4.

12. Howard D, Cordell R, McGowan JE Jr, Packard RM, Scott RD 2nd, Solomon SL; Workshop Group. Measuring the economic costs of antimicrobial resistance in hospital settings: summary of the Centers for Disease Control and Prevention-Emory Workshop. Clin Infect Dis. 2001;33(9):1573-8.

13. Cosgrove SE. The relationship between antimicrobial resistance and patient outcomes: mortality, length of hospital stay, and health care costs. Clin Infect Dis. 2006;42 Suppl 2:S82-9.

14. van de Sande-Bruinsma N, Grundmann H, Verloo D, Tiemersma $\mathrm{E}$, Monen J, Goossens $\mathrm{H}$, et al. Antimicrobial drug use and resistance in Europe. Emerg Infect Dis. 2008;14(11):1722-30.

15. European Antimicrobial Resistance Surveillance System (EARSS). EARSS annual report 2008. Bilthoven: Netherlands Centre for Infectious Disease Control, National Institute for Public Health and the Environment (Rijksinstituut voor Volksgezondheid en Milieu, RIVM); 2009. Available from: http://www.ecdc.europa.eu/en/activities/surveillance/EARSNet/Documents/2008_EARSS_Annual_Report.pdf

16. van Hees BC, de Ruiter E, Wiltink EH, de Jongh BM, Tersmette M. Optimizing use of ciprofloxacin: a prospective intervention study. J Antimicrob Chemother. 2008;61(1):210-3.

17. Willemsen I, Cooper B, van Buitenen C, Winters M, Andriesse $\mathrm{G}$, Kluytmans J. Improving quinolone use in hospitals by using a bundle of interventions in an interrupted time series analysis. Antimicrob Agents Chemother. 2010;54(9):3763-9.

\section{References}

1. Cooke DM, Salter AJ, Phillips I. The impact of antibiotic policy on prescribing in a London teaching hospital. A oneday prevalence survey as an indicator of antibiotic use. I Antimicrob Chemother. 1983;11(5):447-53.

2. Ansari F, Erntell M, Goossens H, Davey P. The European surveillance of antimicrobial consumption (ESAC) pointprevalence survey of antibacterial use in 20 European hospitals in 2006. Clin Infect Dis. 2009;49(10):1496-504.

3. Willemsen I, Groenhuijzen A, Bogaers D, Stuurman A, van Keulen P, Kluytmans J. Appropriateness of antimicrobial therapy measured by repeated prevalence surveys. Antimicrob Agents Chemother. 2007;51(3):864-7. 